\title{
Experimental Observation of Inviscid Burgers' Equation Dynamics in Nonlinear Fiber Optics
}

\author{
Benjamin Wetzel ${ }^{1,2}$, Domenico Bongiovanni ${ }^{1}$, Michael Kues ${ }^{1}$, Yi Hu${ }^{3}$, Zhigang Chen ${ }^{3,4}$, Stefano Trillo , \\ John M. Dudley ${ }^{6}$, Stefano Wabnitz ${ }^{7}$, Roberto Morandotti ${ }^{1}$ \\ 1 Institut National de la Recherche Scientifique, Université du Québec, Varennes, Québec J3X 1S2, Canada \\ 2 School of Mathematical and Physical Sciences, University of Sussex, Sussex House, Falmer, Brighton BN1 9RH, UK \\ 3 TEDA Applied Physics Institute and School of Physics, Nankai University, Tianjin 300457, China \\ 4 Department of Physics \& Astronomy, San Francisco State University, San Francisco, CA 94132, USA \\ 5 Dipartimento di Ingegneria, Università di Ferrara, Via Saragat 1, 44122 Ferrara, Italy \\ 6 Institut FEMTO-ST, UMR 6174 CNRS-Université de Franche-Comté, 25030 Besançon, France \\ 7 Dipartimento di Ingegneria dell'Informazione, Università degli Studi di Brescia, and INO-CNR, via Branze 38, I-25123, Brescia, Italy \\ benjamin.wetzel@emt.inrs.ca
}

\begin{abstract}
We report on the experimental observation of inviscid Burgers' equation dynamics, obtained when a properly tailored pulse propagates in an optical fiber. Experimental results show controllable pulse steepening and shock formation in excellent agreement with theory.

OCIS codes: (060.4370) Nonlinear optics, fibers; (190.5530) Pulse propagation and temporal solitons
\end{abstract}

\section{Introduction}

One of the seminal equations of fluid dynamics is the Inviscid Burgers' Equation (IBE), a one-dimensional analogue of the Navier-Stokes equation in the limit of zero viscosity, which describes nonlinear wave dynamics in incompressible fluids. The IBE has particular importance in understanding the dynamics of shock formation and is thus widely used well beyond hydrodynamics [1] (e.g. to model the formation of large-scale structures in the early universe or even traffic flow evolution). Surprisingly, however, experiments in Burgers' equation systems are often limited by viscosity and dissipation, precluding a quantitative comparison with theory, especially in the limit of evolution towards a shock (the so-called inviscid case). Here, we show that this problem can be solved in an optical system. By properly tailoring an ultrashort pulse injected into a highly nonlinear fiber, propagation can be accurately mapped to an IBE model, allowing quantitative measurements and comparison of pulse front steepening, together with a novel approach to efficiently control shock dynamics. Our results pave the way towards the experimental study, in a convenient benchtop system, of complex physical phenomena otherwise difficult to access.

\section{Theory}

The IBE can be derived from the Euler momentum equation in the absence of pressure gradient and external forces, but interestingly, it can also be obtained from the Nonlinear Schrödinger Equation (NLSE) when considering a pulse of envelope $\mathrm{A}(\mathrm{z}, \mathrm{T})$ propagating in an optical fiber in a weakly (normal) dispersive regime [2]. In this particular case (i.e. when the dispersion length is much longer than the nonlinear length), if an input excitation has the particular form of a simple Riemann pulse $A_{R}$ where the instantaneous frequency profile (chirp) is a properly scaled replica of the amplitude, then this proportionality is preserved during propagation so that $[3,4]$ :

$$
A_{R}(z, T)=\left|A_{R}(z, T)\right| \exp \left[\mp i 2 \sqrt{\frac{\gamma}{\beta_{2}}} \int_{-\infty}^{T}\left|A_{R}\left(z, T^{\prime}\right)\right| d T^{\prime}\right],
$$

where $\gamma$ is the nonlinear Kerr coefficient and $\beta_{2}>0$ the group velocity dispersion of the fiber at the excitation wavelength. Remarkably, under these conditions, the amplitude obeys the propagation dynamics described by the IBE:

$$
\frac{\partial\left|A_{R}\right|}{\partial z} \pm 3 \sqrt{\gamma \beta_{2}}\left|A_{R}\right| \frac{\partial\left|A_{R}\right|}{\partial T}=0
$$

Physically, IBE propagation is associated with pulse envelope steepening and eventually the formation of a socalled gradient catastrophe, where amplitude derivatives tend to infinity and lead to characteristic shock wave behavior $[3,4]$. Importantly, since the IBE can be solved using the method of characteristics (providing a parametric representation of the energy flow) [1], we can analytically predict the distance at which the shock occurs for arbitrary input pulses satisfying the IBE. 


\section{Results}
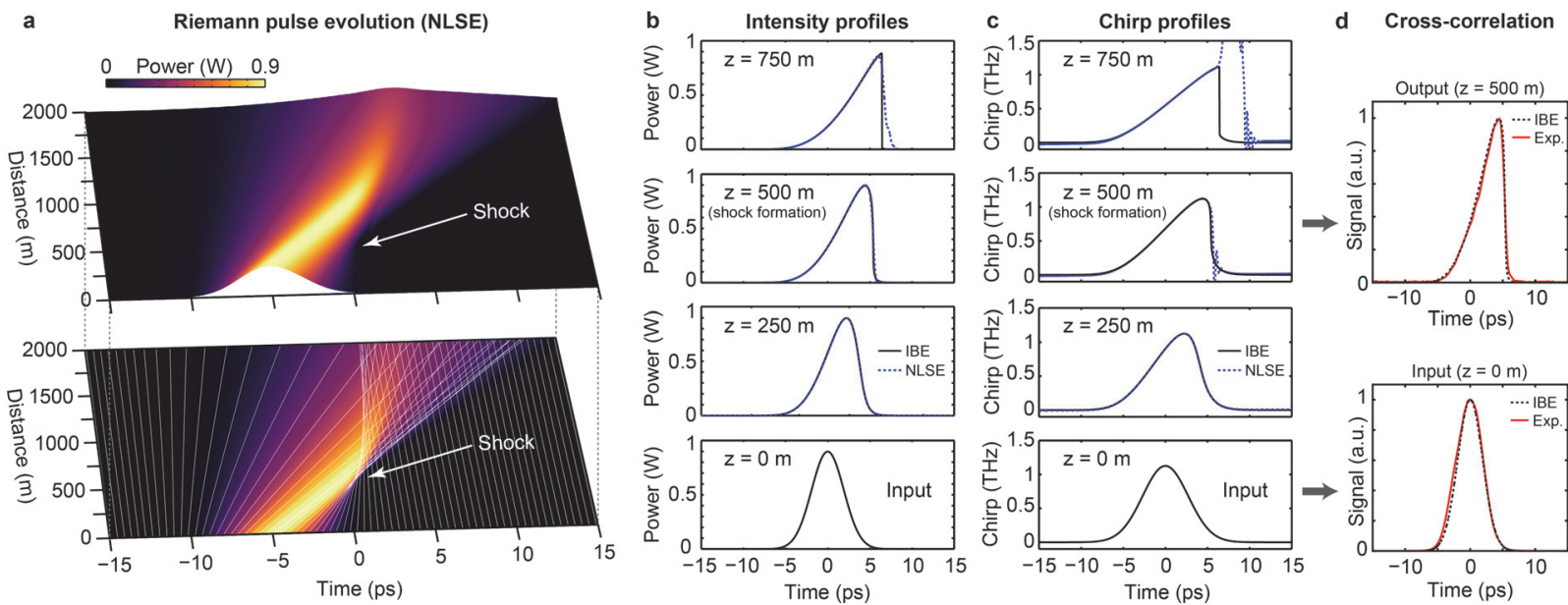

Fig. 1. (a) 3D intensity plot of the NLSE simulations used to model the evolution of a Riemann pulse over $2 \mathrm{~km}$ of fiber (top). The projected pulse intensity (bottom) is compared with the characteristic lines obtained analytically from the IBE (white lines), showing shock formation at $\mathrm{z}=$ $500 \mathrm{~m}$, where the characteristics intersect to give rise to a multi-valued solution (Parameters: Gaussian pulse with $0.89 \mathrm{~W}$ peak power and $4.5 \mathrm{ps}$ duration (FWHM) propagating in a HNLF: $\beta_{2}=0.8397 \mathrm{ps}^{2} \cdot \mathrm{km}^{-1}$ and $\gamma=11.7 \mathrm{~W}^{-1} \cdot \mathrm{km}^{-1}$ at $1550 \mathrm{~nm}$ ). Temporal profiles of (b) intensity and (c) chirp are shown at selected distances, comparing IBE predictions (solid black) with NLSE simulations (dashed blue). (d) Cross-correlation traces are extracted at the input and output of the 500m fiber, comparing experimental results (solid red line) with the profiles expected from IBE simulations (black dashed line)

Numerical results illustrating Riemann pulse propagation in $2 \mathrm{~km}$ of highly nonlinear fiber (HNLF) are shown in Fig. 1(a-c). We see progressive steepening of the pulse trailing edge until $\mathrm{z}=500 \mathrm{~m}$, where a near-vertical front is formed. The calculated IBE characteristic lines shown in Fig. 1a (white lines) follow the direction of the energy flow and the shock formation distance obtained from simulations is elegantly predicted from the IBE (500 $\mathrm{m}$ for our parameters). In essence, until the shock onset, the IBE and NLSE predictions are indistinguishable, illustrating how the Riemann pulse maintains its proportionality between chirp and amplitude throughout propagation. For $\mathrm{z}>500$ $\mathrm{m}$, however, the IBE representation of the NLSE solutions loses validity: chirp oscillations develop on the pulse trailing edge as a typical signature of dispersive shock waves formation [5]. To observe these effects experimentally, a pulse train emitted from an optical parametric oscillator (260 fs duration) is spectrally shaped in intensity and phase (via a commercial Waveshaper) and amplified to generate the desired Riemann pulse (4.5 ps duration, $0.89 \mathrm{~W}$ peak power). The target pulse is then injected into a 500m-long HNLF and characterized at both the input and output using standard techniques (i.e. optical spectrum analyser, cross-correlator/FROG). The experimental results are in excellent agreement with both numerical and analytical predictions as illustrated in Fig. 1(d), where we show the experimentally measured cross-correlation traces at the input and output $(\mathrm{z}=500 \mathrm{~m})$ of the HNLF, compared with those expected from IBE predictions.

Further studies are carried out considering more arbitrary (asymmetric) input Riemann pulses generated through an additional tailoring of the initial pulse spectral phase. Both experimental and numerical results show the ability to efficiently tune the distance at which the shock develops, over several hundreds of meters, by simply varying the degree of asymmetry of the initial pulse.

These results in an optical system therefore provide, we believe, the first clear quantitative and controllable experimental demonstration of IBE dynamics in a wave system. In the framework of nonlinear fiber optics, the ability to transform propagation dynamics from a NLSE system to an IBE model of greatly reduced complexity represents a significant addition to the available experimental toolbox for studying nonlinear dynamics.

\section{References}

[1] J. Bec and K. Khanin, "Burgers turbulence,” Phys. Rep. 447, 1 - 66 (2007).

[2] Y. Kodama and S. Wabnitz, "Analytical theory of guiding-center nonreturn-to-zero and return-to-zero signal transmission in normally dispersion nonlinear optical fibres," Opt. Lett. 20, 2291-2293 (1995).

[3] S. Malaguti, A. Corli and S. Trillo, "Control of gradient catastrophes developing from dark beams. Opt. Lett. 35, 4217-4219 (2010).

[4] S. Wabnitz, "Optical tsunamis: shoaling of shallow water rogue waves in nonlinear fibres with normal dispersion," J. Opt. 15, 064002 (2013);

[5] J. Fatome et al., "Observation of optical undular bores in multiple four-waves mixing," Phys. Rev. X 4, 021022 (2014). 